ROCKY MOUNTAIN

JOURNAL OF MATHEMATICS

Volume 31, Number 4, Winter 2001

\title{
EXTENDED RIEMANN ZETA FUNCTIONS
}

\author{
M. ASLAM CHAUDHRY, ASGHAR QADIR, M.T. BOUDJELKHA, \\ M. RAFIQUE AND S.M. ZUBAIR
}

\begin{abstract}
Analogous to recent useful generalizations of the family of gamma functions and beta functions, extensions of the Riemann zeta function are presented, for which the usual properties and representations are naturally and simply extended. In analogy to these extensions, the extended Hurwitz functions are introduced. Hurwitz-type formulae are also proved.
\end{abstract}

1. Introduction. The zeta function, though originally introduced by Euler, was independently used by Riemann to attack a problem in the theory of prime numbers $[\mathbf{4}],[\mathbf{8}],[\mathbf{1 1}],[\mathbf{1 3}]$. It was known that prime numbers become progressively sparser for large values but no explicit expression explaining how they become so was available until the time of Legendre and Gauss. Writing the number of primes less than or equal to $n$ as

$$
\pi(n)=\sum_{p \leq n} 1
$$

where the summation extends only over the primes. At age 15 Gauss, in 1792 , conjectured that as $n \rightarrow \infty$,

$$
\pi(n) \sim n / \log n
$$

In an attempt to prove the conjecture, Riemann used the zeta function extended to complex variables. (It may be mentioned in passing that though the Riemann proof was incomplete, there is now available an

1991 AMS Mathematics Subject Classification. 33B99, 33C15, 11M06, 11M35, $11 \mathrm{M} 99$.

Key words and phrases. Gamma function, generalized gamma function, zeta functions, integral transforms.

We deeply regret to inform you that the fourth author, M. Rafique, died on 16 June 1996.

Received by the editors on January 23, 2000, and in revised form on July 18, 2000.

Copyright (C)2001 Rocky Mountain Mathematics Consortium 
elementary proof of this result and it is commonly known as the prime number theorem [4], [16]).

Studying the properties of the zeta function, Riemann conjectured that the nontrivial zeros of $\zeta(\alpha), \alpha=\sigma+i \tau$, lie on the critical line $\sigma=1 / 2$ in the complex plane. Though it is proved that they are restricted to the strip, $0<\sigma<1$ (Hardy managed to show that there are infinitely many zeros on the critical line), there is still no proof of the Riemann hypothesis [4], [8], [13].

In this paper, we present two extensions of the Riemann zeta function which are closely related to each other. There being infinitely many new functions and infinitely many extensions or generalizations possible for well-known functions, one needs some clear-cut criteria to determine whether a given extension is worthwhile or not. If it arises in diverse problems, or interesting new relations turn up between it and other functions, or new insights are provided for the original functions, or particularly elegant results can be found for the new function, it would be worthwhile. In our case, we do find natural extensions of the previous results, obtain new results for the extensions and expect that there will be a wider applicability of these extended zeta functions.

Our extensions were motivated by the wide applications of the generalization of the family of gamma functions $[\mathbf{2}],[\mathbf{3}]$ and the beta function [1]. We use integral representations of the zeta function to extend them analogously to the generalized gamma function. We also apply our extension procedure to the Hurwitz zeta function, $\zeta(\alpha, q)$, and obtain the corresponding extensions for it. The asymptotic representations of these functions for small values of the parameter are proved as well. Hurwitz-type formulae for the corresponding loop integrals are also proved.

For clarity of presentation, we state our results as theorems and take $\alpha=\sigma+i \tau$.

2. The extended zeta function $\zeta_{b}(\alpha)$. The original definition of the zeta function by Euler $[\mathbf{1 1}],[\mathbf{1 3}]$ was

$$
\zeta(\sigma)=\sum_{n=1}^{\infty} n^{-\sigma}, \quad \sigma>1 .
$$

It was known that this function has the integral representation 
[13, p. 18]

$$
\zeta(\alpha)=\frac{1}{\Gamma(\alpha)} \int_{0}^{\infty} t^{\alpha-1}\left(1-e^{-t}\right)^{-1} e^{-t} d t
$$

The integral in (2.2) becomes singular for $\sigma \leq 1$ because of the singularity of the integrand at $t=0$. This integrand is similar to that appearing in the Euler gamma function [3] which was generalized to

$$
\Gamma_{b}(\alpha):=\int_{0}^{\infty} t^{\alpha-1} e^{-t-b / t} d t, \quad b>0 ; b=0, \sigma>0 .
$$

This function reduces to the usual gamma function for $b=0$. The corresponding extended zeta function is defined as

$$
\begin{gathered}
\zeta_{b}(\alpha):=\frac{1}{\Gamma(\alpha)} \int_{0}^{\infty} t^{\alpha-1}\left(1-e^{-t}\right)^{-1} e^{-t-b / t} d t \\
b>0 ; \quad b=0, \sigma>1 .
\end{gathered}
$$

It avoids the singularity of the integrand at $t=0$ for $b>0$ as it is exponentially suppressed, see Figure 1 (given in Section 3). As such, it allows us to continue this function into the domain $\sigma<1$. Clearly, in the limit $b \rightarrow 0$ we recover the original zeta function for $\sigma>1$.

\section{Theorem 1.}

$$
\zeta_{b}(\alpha)=\frac{1}{\Gamma(\alpha)} \sum_{n=1}^{\infty} \Gamma_{n b}(\alpha) n^{-\alpha}, \quad b>0 ; b=0, \sigma>1 .
$$

Proof. Expanding $\left(1-e^{-t}\right)^{-1}$ in $(2.4)$ as a power series in $e^{-t}$, we obtain

$$
\zeta_{b}(\alpha)=\frac{1}{\Gamma(\alpha)} \sum_{n=1}^{\infty} \int_{0}^{\infty} t^{\alpha-1} e^{-n t-b / t} d t .
$$

Rescaling the integration variable by $n^{-1}$, we prove the theorem. To justify the inversion of order of summation and integration, it may be noted that the series

$$
\sum_{n=1}^{\infty} t^{\sigma-1} e^{-n t-b / t}
$$


converges to a function which is integrable. But when $b=0$, the assumption $\sigma>1$ is essential to secure convergence of the improper integral

$$
\int_{0}^{\infty} \frac{t^{\sigma-1}}{e^{t}-1} d t
$$

Clearly, as $\Gamma_{0}(\alpha)=\Gamma(\alpha)$, setting $b=0$ in (2.5) yields (2.1).

Corollary. Using the reflection property of the generalized gamma function $[\mathbf{3}]$

$$
\Gamma_{b}(-\alpha)=b^{-\alpha} \Gamma_{b}(\alpha), \quad b>0,
$$

we immediately obtain the relationship

$$
\sum_{n=1}^{\infty} \Gamma_{n b}(\alpha)=b^{\alpha} \Gamma(-\alpha) \zeta_{b}(-\alpha), \quad b>0 .
$$

Exploiting the representation ([7, p. 696])

$$
\Gamma_{b}(\alpha)=2 b^{\alpha / 2} K_{\alpha}(2 \sqrt{b}), \quad b>0,
$$

we can relate the extended zeta function to the Macdonald function by

$$
\zeta_{b}(\alpha)=\frac{2 b^{\alpha / 2}}{\Gamma(\alpha)} \sum_{n=1}^{\infty} n^{-\alpha / 2} K_{\alpha}(2 \sqrt{n b}), \quad b>0 .
$$

\section{Theorem 2.}

$$
\begin{gathered}
\zeta_{b}(\alpha) \Gamma(\alpha)=2^{\alpha-1} \int_{0}^{\infty} t^{\alpha-1} e^{-t-b / 2 t} \operatorname{csch}(t) d t \\
b>0 ; \quad b=0, \sigma>1 .
\end{gathered}
$$

Proof. This result follows by changing $t$ to $2 t$ in (2.4) and using the fact that

$$
\left(e^{2 t}-1\right)^{-1}=e^{-t} \operatorname{csch}(t) / 2 .
$$


Putting $b=0$ gives

$$
\zeta(\alpha) \Gamma(\alpha)=2^{\alpha-1} \int_{0}^{\infty} t^{\alpha-1} e^{-t} \operatorname{csch}(t) d t,
$$

which is a standard result for the Riemann zeta function [5, p. 32(4)].

\section{Theorem 3.}

$$
\begin{aligned}
\zeta_{b}(\alpha)-2^{-\alpha} \zeta_{2 b}(\alpha) & =\frac{1}{\Gamma(\alpha)} \sum_{n=1}^{\infty} \frac{\Gamma_{(2 n-1) b}(\alpha)}{(2 n-1)^{\alpha}} \\
b>0 ; \quad b & =0, \sigma>1 .
\end{aligned}
$$

Proof. Expanding the left-hand side as a summation of the generalized gamma function, we have

$$
\text { LHS }=\frac{1}{\Gamma(\alpha)}\left[\sum_{n=1}^{\infty} \frac{\Gamma_{n b}(\alpha)}{n^{\alpha}}-\frac{1}{2^{\alpha}} \sum_{n=1}^{\infty} \frac{\Gamma_{n(2 b)}(\alpha)}{n^{\alpha}}\right] .
$$

We can combine $2^{\alpha}$ with $n^{\alpha}$ to obtain $(2 n)^{\alpha}$ and express $\Gamma_{2(n b)}$ as $\Gamma_{(2 n) b}$. Thus the right-hand side of (2.14) becomes the difference between the sum over all natural numbers and even natural numbers, which is simply the sum over all odd natural numbers, namely, the right-hand side of (2.13). This proves the result.

Corollary (see [11, p. 36]).

$$
\sum_{n=1}^{\infty}(2 n-1)^{-\alpha}=\left(1-2^{-\alpha}\right) \zeta(\alpha), \quad \sigma>1 .
$$

Proof. This standard result for the zeta function $[\mathbf{5}$, p. 32(3)] is directly obtained by setting $b=0$ in (2.13).

\section{Theorem 4.}

$$
\begin{gathered}
\zeta_{b}(\alpha)-2^{1-\alpha} \zeta_{2 b}(\alpha)=\frac{1}{\Gamma(\alpha)} \sum_{n=1}^{\infty} \frac{(-1)^{n-1} \Gamma_{n b}(\alpha)}{n^{\alpha}} \\
b>0 ; \quad b=0, \sigma>0 .
\end{gathered}
$$


Proof. Expanding the right-hand side of (2.16), we get a summation over odd natural numbers minus that over even natural numbers. Thus,

$$
\operatorname{RHS}=\frac{1}{\Gamma(\alpha)} \sum_{n=1}^{\infty} \frac{\Gamma_{(2 n-1) b}(\alpha)}{(2 n-1)^{\alpha}}-\frac{2^{-\alpha}}{\Gamma(\alpha)} \sum_{n=1}^{\infty} \frac{\Gamma_{n(2 b)}(\alpha)}{n^{\alpha}} .
$$

Now using (2.5), with $b$ replaced by $2 b$ and (2.13), we see that

$$
\mathrm{RHS}=\zeta_{b}(\alpha)-2^{-\alpha} \zeta_{2 b}(\alpha)-2^{-\alpha} \zeta_{2 b}(\alpha)
$$

Simplifying the right-hand side in (2.18), we get the left-hand side of (2.16), thus proving the result.

\section{Corollary.}

$$
\sum_{n=1}^{\infty}(-1)^{n-1} n^{-\alpha}=\left(1-2^{1-\alpha}\right) \zeta(\alpha), \quad \sigma>0 .
$$

Proof. This standard result, [13, p. 21], is directly obtained by setting $b=0$ in $(2.16)$.

\section{Theorem 5.}

$$
\begin{aligned}
\zeta_{b}(\alpha)-2^{1-\alpha} \zeta_{2 b}(\alpha) & =\frac{1}{\Gamma(\alpha)} \int_{0}^{\infty} t^{\alpha-1}\left(1+e^{-t}\right)^{-1} e^{-t-b / t} d t \\
b & >0 ; b=0, \sigma>0 .
\end{aligned}
$$

Proof. Using the definition of the generalized gamma function, given by (2.4), and rescaling the variable of integration $t$ by $n$, we get

$$
n^{-\alpha} \Gamma_{n b}(\alpha)=\int_{0}^{\infty} t^{\alpha-1} e^{-n t-b / t} d t
$$

Whence, multiplying by $(-1)^{n-1}$ and summing both sides over positive natural numbers $n$, we obtain

$$
\sum_{n=1}^{\infty}(-1)^{n-1} \Gamma_{n b}(\alpha) n^{-\alpha}=\int_{0}^{\infty} t^{\alpha-1}\left(1+e^{-t}\right)^{-1} e^{-t-b / t} d t
$$


Using (2.22) and the previous theorem, we arrive at (2.20).

\section{Corollary.}

$$
\begin{aligned}
\zeta_{b}(\alpha)-2^{1-\alpha} \zeta_{2 b}(\alpha) & =\frac{2^{\alpha-1}}{\Gamma(\alpha)} \int_{0}^{\infty} t^{\alpha-1} e^{-t-b / 2 t} \operatorname{sech}(t) d t \\
b & >0 ; \quad b=0, \sigma>0 .
\end{aligned}
$$

Proof. Scaling the variable of integration in (2.20) by 2, directly gives the above result as

$$
\left(1+e^{-2 t}\right)^{-1}=\frac{1}{2} e^{t} \operatorname{sech}(t) .
$$

\section{Corollary.}

$$
\left(1-2^{1-\alpha}\right) \zeta(\alpha)=\frac{2^{\alpha-1}}{\Gamma(\alpha)} \int_{0}^{\infty} t^{\alpha-1} e^{-t} \operatorname{sech}(t) d t, \quad \sigma>0 .
$$

Proof. Obtained directly from (2.23), with $b=0$, is a standard result for the zeta function, see [5, p. 32].

\section{Corollary.}

$$
\zeta(\alpha)=\frac{1}{\Gamma(\alpha)\left(1-2^{1-\alpha}\right)} \int_{0}^{\infty} \frac{t^{\alpha-1}}{1+e^{t}} d t, \quad \sigma>0,
$$

is another form of (2.24), see [5, p. 32]).

3. Transforms and the extended zeta function. It is of particular interest to find relationships between our extended zeta function and transforms of various functions. This may, alternatively, be thought of as the representation of this function in terms of transforms. In particular, the extended zeta function (2.4) can be written as the Mellin transform representation to give

$$
\zeta_{b}(\alpha)=\frac{1}{\Gamma(\alpha)} \mathcal{M}\left\{\frac{e^{-t-b t^{-1}}}{1-e^{-t}} ; \alpha\right\}, \quad b>0 ; \quad b=0, \sigma>1 .
$$


The transformation $t=e^{-x}$ in (2.4) yields

$$
\zeta_{b}(\alpha)=\frac{1}{\Gamma(\alpha)} \int_{-\infty}^{\infty} \frac{\exp \left(-b e^{x}\right)}{\exp \left(e^{-x}\right)-1} e^{-\alpha x} d x
$$

which is the bilateral Laplace transform representation. The substitution $\alpha=\sigma+i \tau$ in (3.2) yields

$$
\zeta_{b}(\alpha)=\frac{1}{\Gamma(\alpha)} \int_{-\infty}^{\infty} \frac{\exp \left(-\sigma x-b e^{x}\right)}{\exp \left(e^{-x}\right)-1} e^{-i \tau x} d x
$$

It can be written in the operational form to give

$$
\zeta_{b}(\alpha)=\frac{1}{\Gamma(\alpha)} F\left\{\frac{\exp \left(-\sigma x-b e^{x}\right)}{\exp \left(e^{-x}\right)-1} ; \tau\right\}
$$

where $F$ is the Fourier transform operator given by

$$
F\{f(x) ; \tau\}=\int_{-\infty}^{\infty} f(x) e^{-i \tau x} d x
$$

\section{Theorem 3.1.}

$$
\int_{0}^{\infty} \zeta_{b}(\alpha) b^{s-1} d b=\frac{\Gamma(s) \Gamma(\alpha+s)}{\Gamma(\alpha)} \zeta(\alpha+s), \quad \sigma>0, \operatorname{Re}(s)>0 .
$$

Proof. For two functions $f(t)$ and $g(t)[6$, p. 308], we have

$$
\mathcal{M}\left\{\int_{0}^{\infty} t^{\alpha-1} f(t) g(\tau / t) d t ; s\right\}=\mathcal{M}\{f(t) ; s+\alpha\} \mathcal{M}\{g(t) ; s\} .
$$

If $f(t)=\left(e^{t}-1\right)^{-1}$ and $g(t)=e^{-t}$, then, $\mathcal{M}\{g(t) ; s\}=\Gamma(s)$ and, in view of (3.1), $\mathcal{M}\{f(t) ; s+\alpha\}=\Gamma(\alpha+s) \zeta(\alpha+s)$. On using (2.4), (3.7) gives

$$
\Gamma(\alpha) \int_{0}^{\infty} \zeta_{\tau}(\alpha) \tau^{s-1} d \tau=\Gamma(s) \Gamma(\alpha+s) \zeta(\alpha+s)
$$


Rearranging and replacing $\tau$ by $b$, we get (3.6).

Remark. The substitution $s=1$ in (3.6) yields an interesting relation

$$
\int_{0}^{\infty} \zeta_{b}(\alpha) d b=\alpha \zeta(\alpha+1), \quad \sigma>0
$$

between the extended and classical zeta function. It shows that there does not exist any $\alpha, \sigma>0$, where the function $\zeta_{b}(\alpha)$ vanishes identically for all $b \geq 0$. If we have $\zeta_{b}\left(\alpha_{0}\right) \equiv 0$ for all $b \geq 0, \sigma_{0}>0$, then it will contradict $(3.9)$ as $\zeta(\alpha+1)$ does not vanish in the region $\sigma>0,[\mathbf{1 3}$, p. 30]. However, it will be interesting to seek the values of $b$ and $\alpha$ for which $\zeta_{b}(\alpha)=0, \sigma>0$.

Theorem 3.2 (Asymptotic representation as $b \rightarrow 0^{+}$).

$$
\begin{aligned}
& \Gamma(\alpha) \zeta_{b}(\alpha) \sim \sum_{n=0}^{\infty} \Gamma(\alpha-n) \zeta(\alpha-n) \frac{(-b)^{n}}{n !}+\Gamma(1-\alpha) b^{\alpha-1} \\
&-\frac{1}{2} \Gamma(-\alpha) b^{\alpha}+b^{\alpha} \sum_{n=0}^{\infty} \frac{\Gamma(-\alpha-2 n-1)}{(2 n+2) !} B_{2 n+2} b^{2 n+1} \\
& 0<\sigma<1, b \rightarrow 0^{+}
\end{aligned}
$$

where $B_{2 n}$ are the Bernoulli numbers [4, p. 14].

Proof. The inverse Mellin transform of (3.6) yields

$$
\begin{gathered}
\Gamma(\alpha) \zeta_{b}(\alpha)=\frac{1}{2 \pi i} \int_{C-i \infty}^{C+i \infty} \Gamma(z) \Gamma(z-\alpha) \zeta(z) b^{\alpha-z} d z \\
0<C<1 .
\end{gathered}
$$

The integrand

$$
F(z):=\Gamma(z) \Gamma(z-\alpha) \zeta(z) b^{\alpha-z}
$$

in (3.11) has three sets of poles, $\{0,1\},\{-2 n-1\}_{n=0}^{\infty}$, and $\{\alpha-n\}_{n=0}^{\infty}$, as the poles of $F$ at $-2 n$ are canceled by the trivial zeros of the zeta 
function. The residues at these poles are given by

$$
\begin{aligned}
\operatorname{Res}\{F ; 0\}= & -\frac{1}{2} \Gamma(-\alpha) b^{\alpha}, \\
\operatorname{Res}\{F ; 1\}= & \Gamma(1-\alpha) b^{\alpha-1}, \\
\operatorname{Res}\{F ;-2 n-1\}= & \frac{(-1)^{2 n+1}}{(2 n+1) !} \Gamma(-2 n-\alpha-1) \\
& \cdot \zeta(-2 n-1) b^{\alpha+2 n+1}, \\
\operatorname{Res}\{F ; \alpha-n\}= & \frac{(-1)^{n}}{n !} \Gamma(\alpha-n) \zeta(\alpha-n) b^{n} .
\end{aligned}
$$

Summing over all these residues yields the asymptotic representation as $b \rightarrow 0^{+}$, see [15, pp. 147-148],

$$
\begin{aligned}
\Gamma(\alpha) \zeta_{b}(\alpha) \sim & {\left[\Gamma(1-\alpha) b^{\alpha-1}-\frac{1}{2} \Gamma(-\alpha) b^{\alpha}\right] } \\
& +\sum_{n=0}^{\infty} \Gamma(\alpha-n) \zeta(\alpha-n) \frac{(-b)^{n}}{n !} \\
& +b^{\alpha} \sum_{n=0}^{\infty} \frac{(-1)^{2 n+1}}{(2 n+1) !} \Gamma(-2 n-\alpha-1) \zeta(-2 n-1) b^{2 n+1} .
\end{aligned}
$$

However, see [4, p. 14],

$$
\zeta(-2 n-1)=\frac{(-1)^{2 n+1}}{2 n+2} B_{2 n+2} .
$$

From (3.17) and (3.18) we arrive at (3.10).

Remark. It follows from (3.10) that as $b \rightarrow 0^{+}$

$$
\zeta_{b}(\alpha)=\zeta(\alpha)+C_{1} b^{\alpha-1}+o(1), \quad 0<\sigma<1 .
$$

It seems disappointing that the function $\zeta_{b}(\alpha)$ does not approximate the zeta function in the critical strip as $b \rightarrow 0^{+}$. However, we have resolved this situation in the second extension of the zeta function. Using the IMSL FORTRAN subroutines for mathematical functions 


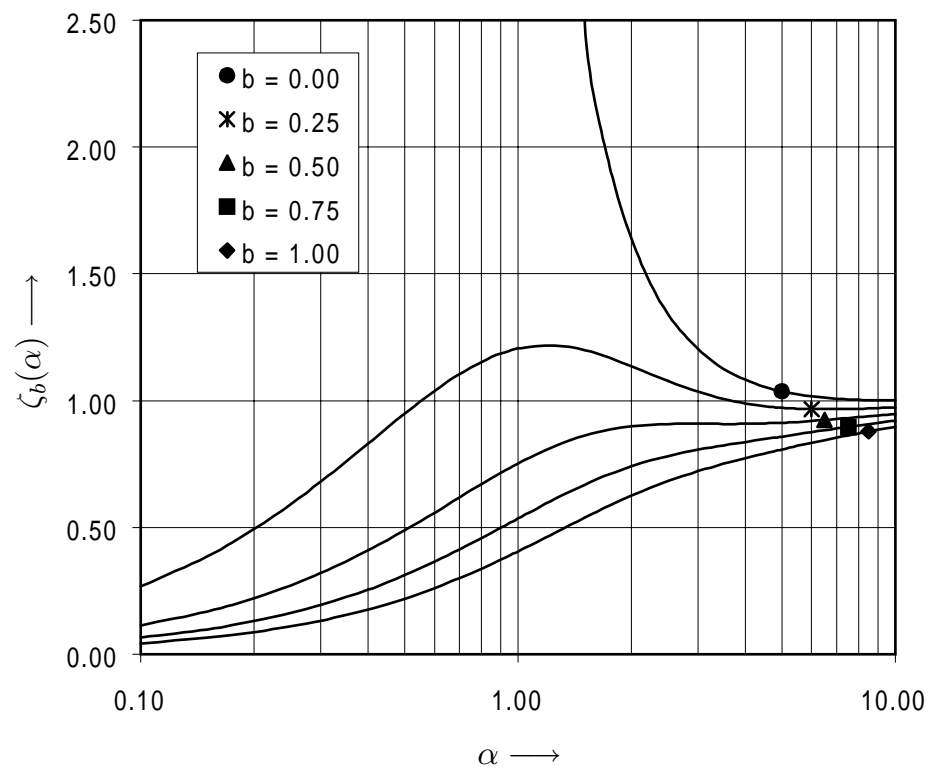

FIGURE 1. Representation of the extended zeta function $\zeta_{b}(\alpha)$.

([12], $[\mathbf{1 0}])$, a graphical representation of the function for $b=0.00$, 0.25, 0.50, 0.75, 1.00 is given in Figure 1.

4. The second extended zeta function $\zeta_{b}^{*}(\alpha)$. The Riemann functional equation $[\mathbf{1 3}$, p. 13]

$$
\zeta(\alpha)=2^{\alpha} \pi^{\alpha-1} \sin \left(\frac{1}{2} \alpha \pi\right) \Gamma(1-\alpha) \zeta(1-\alpha),
$$

and the fact that the zeta function has no zeros in the region $\sigma>1$ implies that all the nontrivial zeros of the zeta function lie in the critical strip, $0<\sigma<1$. The function has simple zeros at $\alpha=$ $-2,-4,-6, \ldots$. Therefore, it is desirable to have an extension of the Riemann zeta function over the region that includes the critical strip as well. Although the function $\zeta_{b}(\alpha)$ extends naturally and simply, the usual properties of the Riemann zeta function for $\sigma>1$, it fails to extend these properties in the critical strip, $0<\sigma<1$. Therefore, it is natural to seek a second extension of the zeta function that carries all the useful properties up to the critical strip. 
In view of (2.20) and (2.26), it seems natural to define the second extended zeta function by

$$
\zeta_{b}^{*}(\alpha):=\frac{1}{C(\alpha)} \int_{0}^{\infty} \frac{t^{\alpha-1} e^{-b / t}}{e^{t}+1} d t, \quad b>0 ; b=0, \sigma>0,
$$

where

$$
C(\alpha):=\Gamma(\alpha)\left(1-2^{1-\alpha}\right) .
$$

It can be seen from (2.26) and (4.2) that

$$
\zeta_{0}^{*}(\alpha)=\zeta(\alpha), \quad \sigma>0 .
$$

Moreover, in view of (2.20) and (4.2), we have an interesting relation

$$
\zeta_{b}^{*}(\alpha)=\frac{\zeta_{b}(\alpha)-2^{1-\alpha} \zeta_{2 b}(\alpha)}{1-2^{1-\alpha}}
$$

between the two extended zeta functions. The relation (4.5) is in fact valid over the whole complex plane by the principle of analytic continuation. Using the IMSL FORTRAN subroutines for mathematical functions $([\mathbf{1 2}],[\mathbf{1 0}])$, a graphical representation of the function for $b=0.00,0.25,0.50,0.75,1.00$ is given in Figures 2 and 3 in the intervals $0<\sigma<1$ and $1<\sigma<10$, respectively.

\section{Theorem (4.1).}

$$
\begin{gathered}
\int_{0}^{\infty} \zeta_{b}^{*}(\alpha) b^{s-1} d b=\frac{\Gamma(s) C(\alpha+s) \zeta(\alpha+s)}{C(\alpha)} s \\
\operatorname{Re}(s)>0, \operatorname{Re}(\alpha)>0
\end{gathered}
$$

Proof. Multiplying both sides in (4.2) by $b^{s-1}$ and integrating from $b=0$ to $b=\infty$, we obtain

$$
\int_{0}^{\infty} \zeta_{b}^{*}(\alpha) b^{s-1} d b=\frac{1}{C(\alpha)} \int_{0}^{\infty} \frac{t^{\alpha-1}}{e^{t}+1}\left(\int_{0}^{\infty} b^{s-1} e^{-b / t} d b\right) d t
$$




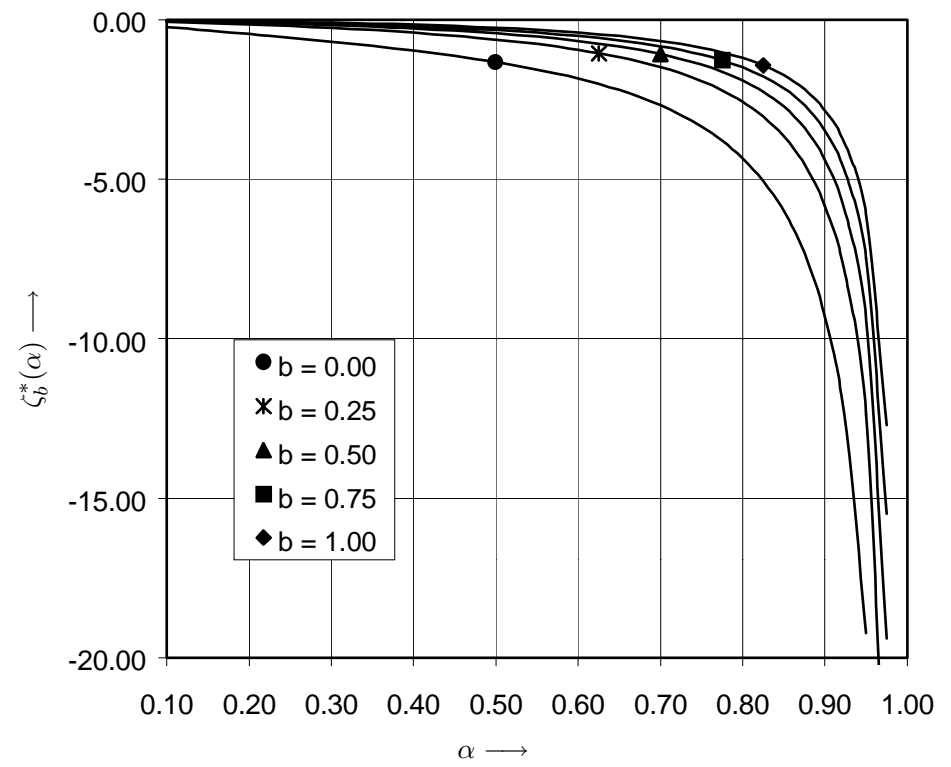

FIGURE 2. Representation of the extended zeta function $\zeta_{b}^{*}(\alpha), 0<\alpha<1$.

The integral

$$
\int_{0}^{\infty} b^{s-1} e^{-b / t} d b
$$

in (4.7) is expressible in terms of the gamma function to give

$$
\int_{0}^{\infty} b^{s-1} e^{-b / t} d b=t^{s} \Gamma(s), \quad \operatorname{Re}(s)>0 .
$$

From (4.7) and (4.8) we get

$$
\int_{0}^{\infty} \zeta_{b}^{*}(\alpha) b^{s-1} d b=\frac{\Gamma(s)}{C(\alpha)} \int_{0}^{\infty} \frac{t^{\alpha+s-1}}{e^{t}+1} d t
$$

which by (2.25) reduces to (4.6).

\section{Corollary.}

$$
\int_{0}^{\infty} \zeta_{b}^{*}(\alpha) d b=C(\alpha+1) \zeta(\alpha+1)
$$




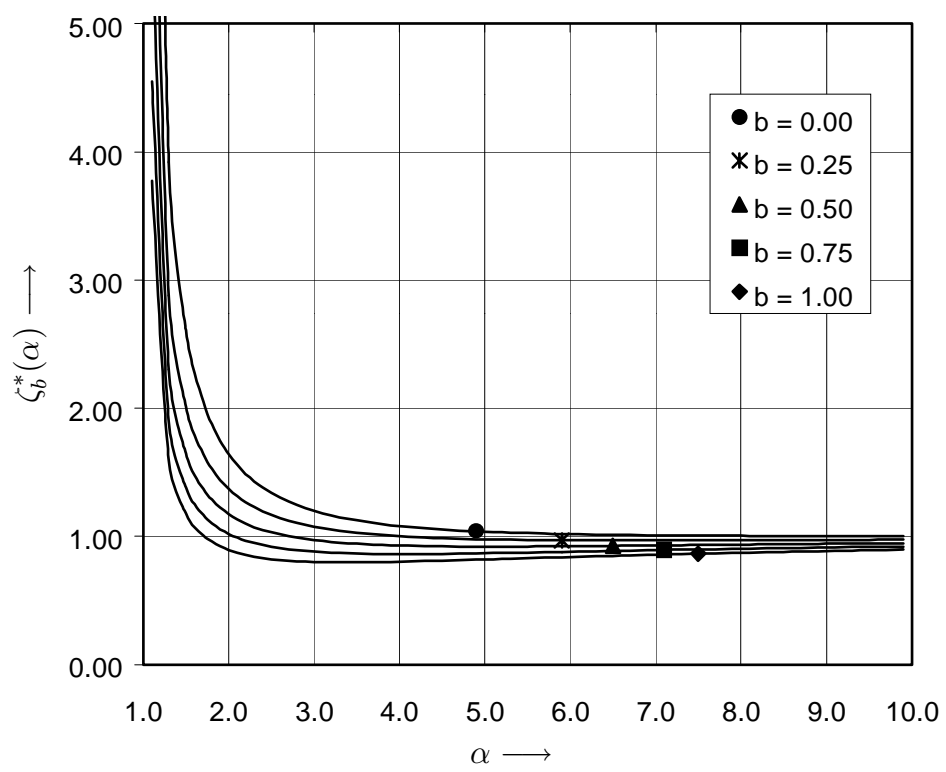

FIGURE 3. Representation of the extended zeta function $\zeta_{b}^{*}(\alpha), 1<\alpha<10$.

Remark. It is to be noted that the extended zeta function (4.2) satisfies the inequality

$$
\left|\zeta_{b}^{*}(\alpha)\right| \leq\left|\frac{C(\sigma)}{C(\alpha)}\right||\zeta(\sigma)|, \quad b>0, \sigma>0 .
$$

One would be interested to seek a constant $K>0$ such that

$$
\left|\zeta_{b}^{*}(\alpha)\right| \leq K|\zeta(\alpha)|, \quad 0<\sigma<1, b \geq 0
$$

The relation (4.10) excludes the existence of such $K>0$ for which (4.12) is true. To prove this we consider the nontrivial zero $\alpha_{0}=$ $\sigma_{0}+i \tau_{0}$ of the zeta function. If (4.12) were true then $\zeta_{b}^{*}\left(\alpha_{0}\right)$ must vanish identically for all $b \geq 0$. But then (4.10) would imply that $C\left(\alpha_{0}+1\right) \zeta\left(\alpha_{0}+1\right)$ should be zero which is not possible as both $C(\alpha)$ and $\zeta(\alpha)$ are different from zero in the region $\sigma>1[\mathbf{1 3}$, p. 2]. 
Theorem 4.2. For $b \rightarrow 0^{+}$

$$
\begin{aligned}
C(\alpha) \zeta_{b}^{*}(\alpha) \sim & \sum_{n=0}^{\infty} C(\alpha-n) \zeta(\alpha-n) \frac{(-b)^{n}}{n !}-\frac{1}{2} \Gamma(-\alpha) b^{\alpha} \\
+ & b^{\alpha} \sum_{n=0}^{\infty} \frac{\left(1-2^{2 n+2}\right)}{(2 n+2) !} \Gamma(-\alpha-2 n-1) B_{2 n+2} b^{2 n+1}, \\
& \quad<\sigma<1,
\end{aligned}
$$

where the $B_{k}$ 's are the Bernoulli numbers.

Proof. This is similar to that of (4.6).

\section{Corollary.}

$$
\zeta_{b}^{*}(\alpha)=\zeta(\alpha)-\frac{\Gamma(-\alpha)}{2 C(\alpha)} b^{\alpha}+O(b), \quad b \rightarrow 0^{+}, 0<\sigma<1 .
$$

Proof. Since the factor $C(\alpha)$ is different from zero in the critical strip, we can divide both sides of (4.13) by $C(\alpha)$ to get (4.14).

\section{Theorem 4.3.}

$$
\zeta_{b}^{*}(\alpha)=\frac{1}{C(\alpha)} \sum_{n=1}^{\infty} \frac{(-1)^{n-1}}{n^{\alpha}} \Gamma_{n b}(\alpha), \quad b>0 ; b=0, \sigma>0 .
$$

Proof. According to (4.2)

$$
\zeta_{b}^{*}(\alpha)=\frac{1}{C(\alpha)} \int_{0}^{\infty} t^{\alpha-1}\left(1+e^{-t}\right)^{-1} e^{-t-b / t} d t .
$$

Replacing the factor $\left(1+e^{-t}\right)^{-1}$ in (4.16) by its geometric series in $e^{-t}$ and then rescaling the variable by $n^{-1}$, we prove (4.15).

\section{Corollary.}

$$
\zeta(\alpha)=\frac{1}{\left(1-2^{1-\alpha}\right)} \sum_{n=1}^{\infty} \frac{(-1)^{n-1}}{n^{\alpha}}, \quad \sigma>0 .
$$


Proof. This result $[\mathbf{1 3}$, p. 21] for the zeta function follows from (4.15) when we substitute $b=0$.

5. Extended Hurwitz zeta functions. The Hurwitz zeta function $\zeta(\alpha, q)$ is initially defined for $\sigma>1$ by the series

$$
\zeta(\alpha, q):=\sum_{n=0}^{\infty} \frac{1}{(n+q)^{\alpha}}, \quad 0<q \leq 1 .
$$

It reduces to $\zeta(\alpha)$ when $q=1$, and to $\left(2^{\alpha}-1\right) \zeta(\alpha)$ when $q=(1 / 2)$, and has the integral representation $([\mathbf{1 3}$, p. 37], $[\mathbf{1 4}$, p. 265])

$$
\zeta(\alpha, q):=\frac{1}{\Gamma(\alpha)} \int_{0}^{\infty} \frac{t^{\alpha-1} e^{-q t}}{1-e^{-t}} d t, \quad \sigma>1,0<q \leq 1 .
$$

In analogy to (2.4) and (4.2), the extended Hurwitz zeta functions $\zeta_{b}(\alpha, q)$ and $\zeta_{b}^{*}(\alpha, q)$ are defined by

$$
\begin{aligned}
\zeta_{b}(\alpha, q) & :=\frac{1}{\Gamma(\alpha)} \int_{0}^{\infty} \frac{t^{\alpha-1} e^{-q t-b / t}}{1-e^{-t}} d t, \\
\sigma & >1,0<q \leq 1, b \geq 0
\end{aligned}
$$

and

$$
\begin{aligned}
\zeta_{b}^{*}(\alpha, q) & :=\frac{1}{C(\alpha)} \int_{0}^{\infty} \frac{t^{\alpha-1} e^{-q t-b / t}}{1+e^{-t}} d t \\
\sigma & >0,0<q \leq 1, b \geq 0,
\end{aligned}
$$

where $C(\alpha)$ is as in (4.3).

It is to be noted that

$$
\zeta_{0}(\alpha, q)=\zeta(\alpha, q), \quad \sigma>1
$$

and

$$
\zeta_{0}^{*}(\alpha, q)=\frac{2^{1-\alpha} \zeta(\alpha, q / 2)-\zeta(\alpha, q)}{1-2^{1-\alpha}}, \quad \sigma>0 .
$$

The relation (5.6) can be extended to an arbitrary $b$ with $b \geq 0$ as follows. 


\section{Theorem 5.1.}

$$
\zeta_{b}^{*}(\alpha, q)=\frac{2^{1-\alpha} \zeta_{2 b}(\alpha, q / 2)-\zeta_{b}(\alpha, q)}{1-2^{1-\alpha}} .
$$

Proof. Replacing $q$ by $q / 2$ in (5.3) and changing the variable of integration from $t$ to $2 t$, we get an expression for $\zeta_{2 b}(\alpha, q / 2)$, i.e.,

$$
\zeta_{2 b}(\alpha, q / 2)=\frac{2^{\alpha}}{\Gamma(\alpha)} \int_{0}^{\infty} \frac{t^{\alpha-1} e^{-q t-b / t}}{1-e^{-2 t}} d t .
$$

However,

$$
2\left(1-e^{-2 t}\right)^{-1}=\left(1-e^{-t}\right)^{-1}+\left(1+e^{-t}\right)^{-1} .
$$

From (5.8) and (5.9) we get

$$
2^{1-\alpha} \zeta_{2 b}(\alpha, q / 2)-\zeta_{b}(\alpha, q)=\left(1-2^{1-\alpha}\right) \zeta_{b}^{*}(\alpha, q) .
$$

Dividing by the factor $1-2^{1-\alpha}$ we obtain (5.7).

Remark. It is important to note that the continuous analogue to the classical relation, see [13, p. 37],

$$
\zeta\left(\alpha, \frac{1}{2}\right)=\left(2^{\alpha}-1\right) \zeta(\alpha)
$$

is

$$
\zeta_{b}\left(\alpha, \frac{1}{2}\right)=2^{\alpha} \zeta_{b / 2}(\alpha)-\zeta_{b}(\alpha)
$$

Moreover, substituting $q=1$ in (5.7) and using (5.2) we arrive at (4.5).

Besides the fact that the substitution $b=0$ in (5.3) yields the Hurwitz zeta function, it is important to see that these functions are related via the Mellin transform. 


\section{Theorem 5.2.}

$$
\begin{gathered}
\int_{0}^{\infty} \zeta_{b}(\alpha, q) b^{s-1} d b=\frac{\Gamma(s) \Gamma(\alpha+s) \zeta(\alpha+s, q)}{\Gamma(\alpha)}, \\
\operatorname{Re}(s)>0, \operatorname{Re}(\alpha+s)>1
\end{gathered}
$$

Proof. Multiplying both sides in (5.3) with $b^{s-1}$ and integrating with respect to $b$ from $b=0$ to $b=\infty$, and using the Fubini theorem we get

$$
\begin{array}{rl}
\int_{0}^{\infty} \zeta_{b}(\alpha, q) b^{s-1} & d b \\
& =\frac{1}{\Gamma(\alpha)} \int_{0}^{\infty} \frac{t^{\alpha-1} e^{-q t}}{1-e^{-t}}\left(\int_{0}^{\infty} b^{s-1} e^{-b / t} d b\right) d t
\end{array}
$$

The integral in (5.14) is solvable in terms of the gamma function to give

$$
\int_{0}^{\infty} b^{s-1} e^{-b / t} d b=t^{s-1} \Gamma(s), \quad \operatorname{Re}(s)>0 .
$$

From (5.14) and (5.15) we obtain

$$
\int_{0}^{\infty} \zeta_{b}(\alpha, q) b^{s-1} d b=\frac{\Gamma(s)}{\Gamma(\alpha)} \int_{0}^{\infty} \frac{t^{\alpha+s-1} e^{-q t}}{1-e^{-t}} d t,
$$

which is exactly (5.13) when $\operatorname{Re}(\alpha+s)>1$.

\section{Corollary.}

$$
\int_{0}^{\infty} \zeta_{b}(\alpha, q) d b=\alpha \zeta(\alpha+1, q)
$$

Proof. Follows from (5.13) when we substitute $s=1$.

\section{Corollary.}

$$
\begin{gathered}
\Gamma(\alpha) \zeta_{b}(\alpha, q)=\frac{1}{2 \pi i} \int_{C-i \infty}^{C+i \infty} \Gamma(z) \Gamma(z-\alpha) \zeta(z, q) b^{\alpha-z} d z \\
0<C<1
\end{gathered}
$$


Proof. This follows from (5.13) by inverting the Mellin transform. In particular, the substitution $q=1$ in (5.18) yields (3.6).

Remark. In analogy to (5.13), we note that

$$
\begin{gathered}
\int_{0}^{\infty} \zeta_{b}^{*}(\alpha, q) b^{s-1} d b=\frac{\Gamma(s) C(\alpha+s) \zeta_{0}^{*}(\alpha+s, q)}{C(\alpha)}, \\
\operatorname{Re}(s)>0, \operatorname{Re}(\alpha+s)>0,
\end{gathered}
$$

which yields (4.6) when we substitute $q=1$.

Inverting (5.19) we obtain

$$
\begin{gathered}
C(\alpha) \zeta_{b}^{*}(\alpha, q)=\frac{1}{2 \pi i} \int_{C-i \infty}^{C+i \infty} C(z) \Gamma(z-\alpha) \zeta_{0}^{*}(z, q) b^{\alpha-z} d z \\
0<C<1,
\end{gathered}
$$

where $C(\alpha)$ is as defined in (4.3).

6. Extended Hurwitz formula. For the generalized zeta function, the Hurwitz formula [13, p. 37],

$$
\begin{gathered}
\zeta(\alpha, q)=\frac{2 \Gamma(1-\alpha)}{(2 \pi)^{1-\alpha}}\left\{\sin \left(\frac{1}{2} \alpha \pi\right) \sum_{n=1}^{\infty} \frac{\cos 2 \pi q n}{n^{1-\alpha}}\right. \\
\left.+\cos \left(\frac{1}{2} \alpha \pi\right) \sum_{n=1}^{\infty} \frac{\sin 2 \pi q n}{n^{1-\alpha}}\right\} \\
\sigma<0,0<q \leq 1,
\end{gathered}
$$

applies; and the Riemann functional equation

$$
\zeta(\alpha)=2 \frac{\Gamma(1-\alpha)}{(2 \pi)^{1-\alpha}} \sin \left(\frac{1}{2} \alpha \pi\right) \zeta(1-\alpha),
$$

is obtained by putting $q=1$. It seems natural to seek Hurwitz type formulae for the extended zeta functions which would include, as a special case, the classical Hurwitz formula when $b=0$. For this purpose, a review of the analysis by which this formula was obtained, 
see $[\mathbf{1 4}$, p. 226], shows that $\zeta(\alpha, q)$ can be expressed as a Hankel contour integral

$$
\zeta(\alpha, q)=-\frac{\Gamma(1-\alpha)}{2 \pi i} \int_{\infty}^{(0+)}(-z)^{\alpha-1} \frac{e^{-q z}}{1-e^{-z}} d z
$$

and that the contribution of the integral along the small circle $\gamma_{\rho}=$ $\{z:|z|=\rho\}$ tends to zero as the radius $\rho \rightarrow 0$.

By analogy, we consider the loop integral

$$
\int_{\infty}^{(0+)}(-z)^{\alpha-1} \frac{e^{-q z-b / z}}{1-e^{-z}} d z
$$

which can be written as the sum

$$
\begin{aligned}
\int_{\infty}^{(0+)}(-z)^{\alpha-1} & \frac{e^{-q z-b / z}}{1-e^{-z}} d z \\
= & \int_{\gamma_{\rho}}(-z)^{\alpha-1} \frac{e^{-q z-b / z}}{1-e^{-z}} d z+\left(e^{\pi i(\alpha-1)}-e^{-\pi i(\alpha-1)}\right) \\
& \cdot \int_{\rho}^{\infty} x^{\alpha-1} \frac{e^{-q x-b / x}}{1-e^{-x}} d x .
\end{aligned}
$$

It is to be noted that, if $b>0$, the contribution from the integral over the small circle will not vanish as $\rho \rightarrow 0$. In fact, this integral diverges, as $\rho \rightarrow 0$. In view of the above considerations, we introduce the following two related functions

$$
I_{b}(\alpha, q):=-\frac{\Gamma(1-\alpha)}{2 \pi i} \int_{\infty}^{(0+)}(-z)^{\alpha-1} \frac{e^{-q z-b / z}}{1-e^{-z}} d z
$$

and

$$
\begin{gathered}
I_{b}^{*}(\alpha, q):=-\frac{\Gamma(1-\alpha)}{2 \pi i} \int_{\infty}^{(0+)}(-z)^{\alpha-1} \frac{e^{-q z-b / z}}{1+e^{-z}} d z \\
0<q \leq 1, b \geq 0
\end{gathered}
$$

where

$$
\int_{\infty}^{(0+)}
$$


denotes a Hankel contour not containing any of the poles of the integrand, and obtain their corresponding Hurwitz type formulae.

\section{Theorem 6.1.}

$$
\begin{gathered}
I_{b}(\alpha, q)=2 \Gamma(1-\alpha) \sum_{k=1}^{\infty}(2 k \pi)^{\alpha-1} \sin \left(2 k \pi q+\frac{\alpha \pi}{2}-\frac{b}{2 k \pi}\right) \\
\sigma<0,0<q \leq 1, b \geq 0 .
\end{gathered}
$$

Proof. Let

$$
f(z)=(-z)^{\alpha-1} \frac{e^{-q z-b / z}}{1-e^{-z}}, \quad \operatorname{Re} \alpha=\sigma<0,0<q \leq 1, b \geq 0
$$

and consider the contour integral

$$
\frac{1}{2 \pi i} \int_{C_{n}} f(z) d z
$$

where

$$
C_{n}=\mathcal{L}_{n} \cup \Gamma_{n} .
$$

$\Gamma_{n}$ consists of a large circle:

$$
z=R_{n} e^{i \theta}, \quad R_{n}=(2 n+1) \pi, \quad-\pi \leq \theta \leq \pi .
$$

It starts at the point $z=-(2 n+1) \pi e^{i \pi}$, encircles the origin in the clockwise direction and ends at the point $z=-(2 n+1) \pi e^{-i \pi}$. The contour $\mathcal{L}_{n}$ is a loop around the positive real axis. It starts at $z=-(2 n+1) \pi e^{-i \pi}$, goes inward on the upper edge of the "cut" to the point $z=\rho<\pi$, encircles the origin once in the counterclockwise direction and returns along the lower edge of the "cut" to the point $z=-(2 n+1) \pi e^{i \pi}$.

Between $\mathcal{L}_{n}$ and $\Gamma_{n}$, the function $f$ has simple poles at $z= \pm 2 k \pi i$ for nonzero natural numbers $k$. The sum of the residues for the symmetric 
$k$ th poles is

$$
\begin{aligned}
\operatorname{Res}\{f ; 2 k \pi i\}+ & \operatorname{Res}\{f ;-2 k \pi i\} \\
= & (2 k \pi)^{\alpha-1}\left\{e^{i[2 k \pi q+(\alpha-1)(\pi / 2)-(b / 2 k \pi)]}\right. \\
& \left.\quad+e^{-i[2 k \pi q+(\alpha-1)(\pi / 2)-(b / 2 k \pi)]}\right\} \\
= & 2(2 k \pi)^{\alpha-1} \sin \left(2 k \pi q+\alpha \frac{\pi}{2}-\frac{b}{2 k \pi}\right) .
\end{aligned}
$$

By the residue theorem,

(6.8) $-\frac{1}{2 \pi i} \int_{C_{n}} f(z) d z=2 \sum_{k=1}^{n}(2 k \pi)^{\alpha-1} \sin \left(2 k \pi q+\alpha \frac{\pi}{2}-\frac{b}{2 k \pi}\right)$.

To show that the contribution from the large circle tends to zero as $n \rightarrow \infty$, we first note that

$$
\left|\frac{e^{-q z}}{1-e^{-z}}\right| \leq K
$$

on the circle $z=R_{n} e^{i \theta},-\pi \leq \theta \leq \pi$ and $\left|z^{\alpha-1}\right|=\left|R_{n}^{\sigma-1} e^{-\tau \arg (z)}\right| \leq$ $R_{n}^{\sigma-1} e^{|\tau| \pi}$. Therefore, when $\sigma<0$, see [7, p. 968],

$$
\begin{aligned}
\mid \frac{1}{2 \pi i} \int_{\Gamma_{n}}(-z)^{\alpha-1} & \frac{e^{-q z-b / z}}{1-e^{-z}} d z \mid \\
\leq & \frac{K}{2 \pi} \int_{-\pi}^{\pi} R_{n}^{\sigma} e^{|\tau| \pi} e^{-\left(b / R_{n}\right) \cos \theta} d \theta \\
& =K e^{|\tau| \pi} R_{n}^{\sigma} I_{0}\left(\frac{b}{R_{n}}\right) \longrightarrow 0 \quad(\text { as } n \rightarrow \infty) .
\end{aligned}
$$

where $I_{0}(x)$ is the modified Bessel function of order zero, having limit one as $x \rightarrow 0$. Thus, letting $n \rightarrow \infty$ in (6.8) and using (6.9), we arrive at (6.6).

Remark. The substitution $b=0$ in (6.6) yields the Hurwitz formula (6.1). 


\section{Theorem 6.2.}

$$
\begin{aligned}
I_{b}^{*}(\alpha, q)= & 2 \Gamma(1-\alpha) \sum_{k=0}^{\infty}((2 k+1) \pi)^{\alpha-1} \\
& \cdot \sin \left((2 k+1) \pi q+\alpha \frac{\pi}{2}-\frac{b}{(2 k+1) \pi}\right) \\
& \sigma<0,0<q \leq 1, b \geq 0 .
\end{aligned}
$$

Proof. Let

$$
f(z):=(-z)^{\alpha-1} \frac{e^{-q z-b / z}}{1+e^{-z}}, \quad \operatorname{Re} \alpha=\sigma<0,0<q \leq 1, \beta \geq 0,
$$

and consider the contour integral

$$
\frac{1}{2 \pi i} \int_{C_{n}} f(z) d z
$$

where $C_{n}=\mathcal{L}_{n} \cup \Gamma_{n} . \Gamma_{n}$ consists of a large circle: $z=R_{n} e^{i \theta}, R_{n}=2 n \pi$, $-\pi \leq \theta \leq \pi$, and $\mathcal{L}_{n}$ is a similar loop around the positive real axis starting at $z=-2 n \pi e^{-i \pi}$ and ending on the lower edge of the "cut" at the point $z=-2 n \pi e^{i \pi}$. Inside $C_{n}$, the function $f$ has simple poles at $z= \pm(2 k+1) \pi i$. The sum of the residues of the symmetric $k$ th poles is:

$$
\begin{aligned}
& \operatorname{Res}\{f ;(2 k+1) \pi i\}+ \operatorname{Res}\{f ;-(2 k+1) \pi i\} \\
&=[(2 k+1) \pi]^{\alpha-1}\left\{e^{i[(2 k+1) \pi q+(\alpha-1)(\pi / 2)-(b /(2 k+1) \pi)]}\right. \\
&\left.+e^{-i[(2 k+1) \pi q+(\alpha-1)(\pi / 2)-b /((2 k+1) \pi)]}\right\} \\
&=2[(2 k+1) \pi]^{\alpha-1} \sin \left((2 k+1) \pi q+\alpha \frac{\pi}{2}-\frac{b}{(2 k+1) \pi}\right) .
\end{aligned}
$$

By the residue theorem,

$$
\begin{aligned}
& -\frac{1}{2 \pi i} \int_{C_{n}} f(z) d z \\
& \quad=2 \sum_{k=0}^{n-1}[(2 k+1) \pi]^{\alpha-1} \sin \left((2 k+1) \pi q+\alpha \frac{\pi}{2}-\frac{b}{(2 k+1) \pi}\right) .
\end{aligned}
$$


In the contour integral, we now show that the contribution from the large circle tends to zero as $n \rightarrow \infty$. On the circle, $z=R_{n} e^{i \theta}$, $R_{n}=2 n \pi,-\pi \leq \theta \leq \pi$,

$$
\left|\frac{e^{-q z}}{1+e^{-z}}\right| \leq M
$$

a positive constant, and $\left|z^{\alpha-1}\right| \leq R_{n}^{\sigma-1} e^{|\tau| \pi}$. Since $\sigma<0$, we have as $n \rightarrow \infty$,

$$
\begin{aligned}
\mid \frac{1}{2 \pi i} \int_{\Gamma_{n}}(-z)^{\alpha-1} & \frac{e^{-q z-b / z}}{1+e^{-z}} d z \mid \\
& \leq \frac{M}{2 \pi} \int_{-\pi}^{\pi} R_{n}^{\sigma} e^{|\tau| \pi} e^{-\left(b / R_{n}\right) \cos \theta} d \theta \longrightarrow 0
\end{aligned}
$$

By letting $n \rightarrow \infty$ in (6.12) and using (6.9), we arrive at (6.10).

7. The functions $F_{b}(\alpha)$ and $F_{b}^{*}(\alpha)$. In this section we define two functions

$$
F_{b}(\alpha):=\frac{\zeta_{b}(\alpha) \zeta_{2 b}(1-\bar{\alpha})}{\zeta_{b}(1-\bar{\alpha}) \zeta_{2 b}(\alpha)}, \quad 0<\sigma<1, b>0
$$

and

$$
F_{b}^{*}(\alpha):=\frac{\zeta_{b}^{*}(\alpha) \zeta_{2 b}^{*}(1-\bar{\alpha})}{\zeta_{b}^{*}(1-\bar{\alpha}) \zeta_{2 b}^{*}(\alpha)}, \quad 0<\sigma<1, b>0
$$

The asymptotic representation (3.19) yields

$$
F_{b}(\alpha)=2^{1-2 \sigma}\left[1+O\left(b^{\min (\sigma, 1-\sigma)}\right)\right], \quad 0<\sigma<1, b \rightarrow 0^{+},
$$

and this tends to $2^{1-2 \sigma}$ uniformly as $b \rightarrow 0^{+}$.

The asymptotic representation (4.14) yields

$$
\lim _{b \rightarrow 0^{+}} F_{b}^{*}(\alpha)= \begin{cases}1 & \text { if } \zeta(\alpha) \neq 0, \\ 2^{1-2 \sigma} & \text { if } \zeta(\alpha)=0 .\end{cases}
$$


To prove (7.4), we note that $\zeta_{b}^{*}(\alpha)$ converges uniformly to $\zeta(\alpha)$ in $0<\sigma_{0} \leq \sigma \leq \sigma_{1}<1$, as $b \rightarrow 0^{+}$. Thus, if $\zeta(\alpha)$ does not vanish at $\alpha$, the right-hand side in (7.2) would converge to one when $b \rightarrow 0^{+}$. When $\alpha$ is a zero of the zeta function we use (4.14) to evaluate the limit in (7.4) that yields $2^{1-2 \sigma}$.

The limit in (7.4) is important. If an alternate evaluation of the limit shows that

$$
\lim _{b \rightarrow 0^{+}} F_{b}^{*}(\alpha)=1, \quad 0<\sigma<1,
$$

then (7.5) would imply

$$
2^{1-2 \sigma}=1 \quad \text { when } \zeta(\alpha)=0, \quad 0<\sigma<1,
$$

i.e., the nontrivial zeros of the zeta function lie on the critical line $\sigma=1 / 2$. This would prove the Riemann hypothesis and become one of the most important applications of these extended zeta functions.

8. Discussion and conclusion. We have seen that the generalization of the incomplete and complete gamma functions $[\mathbf{2}],[\mathbf{3}]$ and the beta function $[\mathbf{1}]$ is useful for extending the zeta function. However, the extension does not continue to match the zeta function for all ranges of the real part of the argument. The first extension carries on beyond the singularity (at $\sigma=1$ ) on the positive side, while the zeta function is negative for $0<\sigma<1$. Since the extension was designed to be nonsingular at $\sigma=1$ it could not match the zeta function beyond that value.

To extend into the range $0<\sigma<1$, which is of special interest on account of the Riemann hypothesis (that the nontrivial zeros of his zeta function lie on the line $\sigma=1 / 2$, we started with an integral representation of the zeta function valid for $0<\sigma<\infty$ and used the "regularizer" to extend it. The singularity at $\sigma=0$ (for a real argument) was retained by a factor outside the integral. This second extension carries many of the previous properties into the strip $0<\sigma<1$ and has elegant relations to the zeta function itself and its first extension.

There are infinitely many possible extensions of the zeta function. Of course some results analogous to those for the zeta and generalized 
zeta functions would hold for any extension. What we require is that the results be naturally and simply extended. This criterion is met by these extensions. It is expected that such natural extensions would be found useful in answering some of the classical problems.

Acknowledgments. The authors are most grateful to Professor D.R. Heath-Brown for extremely fruitful comments. In particular, the second extension of the zeta function was inspired by his comment. The authors are also indebted to King Fahd University of Petroleum and Minerals, Dhahran, Saudi Arabia for excellent research facilities. M. Aslam Chaudhry is particularly thankful to Professor Zagier for his several private correspondences regarding the results $(3.11),(4.11),(7.3)$, and (7.4). Also, M. Aslam Chaudhry and S.M. Zubair acknowledge the support provided by the University through the research project MS/CLASS/208.

\section{REFERENCES}

1. M. Aslam Chaudhry, Asghar Qadir, M. Rafique and S.M. Zubair, Extension of Euler's beta function, J. Comput. Appl. Math. 78 (1997), 19-32.

2. M. Aslam Chaudhry and S.M. Zubair, Generalized incomplete gamma functions with applications, J. Comput. Appl. Math. 55 (1994), 99-124.

3. - Extended gamma and digamma functions, Fractional Calculus Appl. Anal. 4 (2001), 303-324.

4. H.M. Edwards, Riemann's zeta function, Academic Press, New York, 1974.

5. A. Erdélyi, W. Magnus, F. Oberhettinger and F.G. Tricomi, Higher transcendental functions, Vol. I, McGraw-Hill Book Company, Inc., New York, 1953.

6. - Tables of integral transforms, Vol. II, McGraw-Hill Book Company, Inc., New York, 1954.

7. I.S. Gradshteyn and I.M. Ryzhik, Table of integrals, series and products, 5th ed., English translation from Russian by Scripta Technica, Inc. (Alan Jeffrey, ed.), Academic Press Inc., New York, 1980.

8. G.H. Hardy and J.E. Littlewood, Contributions to the theory of the Riemann zeta functions and the theory of the distribution of primes, Acta Math. 41 (1918), 119-196.

9. D.R. Heath-Brown, private communication, 1996.

10. IMSL, MATH/LIBRARY, Vol. 2, IMSL, Inc., Houston, TX, 1991.

11. A.A. Karatsuba and S.M. Voronin, The Riemann zeta function, translated from Russian by Neal Koblitz, Walter de Gruyter and Co., Berlin, 1992.

12. R.E. Piessens, et al., QUAD-PACK, Springer-Verlag, New York, 1983. 
13. E.C. Titchmarsh, The theory of the Riemann zeta function, Oxford University Press, London, 1957.

14. E.T. Whittaker and G.N. Watson, A course of modern analysis, 4th ed., Cambridge University Press, 1963.

15. R. Wong, Asymptotic approximation of integrals, Academic Press, Boston, 1989.

16. D.B. Zagier, Newman's short proof of the prime number theorem, Amer. Math. Monthly 104 (1997), 705-708.

Department of Mathematical Sciences, King Fahd University of Petroleum and Minerals, Dhahran 31261, Saudi Arabia

E-mail address: maslam@kfupm.edu.sa

Department of Mathematics, Quaid-I-Azam University, Islamabad, Pakistan, and Senior Associate of Abdus Salam Centre for Theoretical Physics, Trieste, Italy

E-mail address: aqadirs@comsats.net.pk

Department of Mathematical Sciences, King Fahd University of Petroleum and Minerals, Dhahran 31261, Saudi Arabia

E-mail address: mtidani@kfupm.edu.sa

Formerly: Department of Mathematical Sciences, King Fahd University of Petroleum and Minerals, Dhahran 31261, Saudi Arabia

Department of Mechanical Engineering, King Fahd University of Petroleum and Minerals, Dhahran 31261, Saudi Arabia

E-mail address: smzubair@kfupm.edu.sa 